Instituto Internacional de Investigación y Desarrollo Tecnológico Educativo INDTEC, C.A.

DOI: https://doi.org/10.29394/scientific.issn.2542-2987.2017.2.6.11.217-237

OAI-PMH: http://www.indteca.com/ojs/index.php/Revista Scientific/oai

\title{
Programa para padres en el Uso de Internet de sus Hijos
}

\author{
Autora: María Viscencia Gil \\ Instituto Tecnológico Antonio José de Sucre, ITAJS \\ mariagil t@hotmail.com \\ Lara, Venezuela
}

\section{Resumen}

El estudio se desarrolló con el propósito de elaborar un programa sobre el ejercicio de la Autoridad-Afecto, dirigido a los padres para el establecimiento de normas en el uso del Internet de sus hijos de la Unidad Educativa Departamento Libertador en Barquisimeto Estado Lara. La investigación fue de tipo proyectiva de campo de carácter descriptivo. La población fue de 60 padres encuestados con un cuestionario dicotómico con respuestas de alternativas Sí y No. La validez se efectuó a través de la técnica de juicios de expertos, para la validación del instrumento y la confiabilidad Kuder Richardson su resultado fue 0,8377 (alta confiabilidad). Asimismo, se empleó la estadística descriptiva mediante la técnica porcentual, los valores fueron analizados en función de las respuestas emitidas por los sujetos en estudio. La presentación se realizó a través de cuadros por dimensiones y gráficos de barra. Entre los hallazgos se encontraron: los padres encuestados no manejan la autoridad, ni se comunican de manera operativa con sus hijos, prestándole poca atención a la parte afectiva; también se evidencia falta de ejercicio Autoridad-Afecto como nivel jerárquico para elaborar normas en el uso de Internet de sus hijos. Estos resultados ameritaron diseñar el establecido programa.

Palabras clave: programa de enseñanza; padre; internet; niño. 


\title{
Program for Parents in the Use of the Internet of their Children
}

\begin{abstract}
The study was developed with the purpose of elaborating a program on the exercise of the Authority-Affect, directed to the parents for the establishment of norms in the use of the Internet of their children of the Educative Unit Department Libertador in Barquisimeto State Lara. The research was of projective type of field of descriptive character. The population was 60 parents surveyed with a dichotomous questionnaire with answers of alternatives Yes and No. The validity was made through the technique of expert judgments, for instrument validation and reliability Kuder Richardson his score was 0.8377 (high reliability). Likewise, descriptive statistics were used by means of the percentage technique, the values were analyzed according to the responses issued by the subjects under study. The presentation was made through tables by dimensions and bar graphs. Among the findings were: parents surveyed do not handle authority, nor communicate in an operative way with their children, paying little attention to the affective part; there is also evidence of lack of Authority-Affect exercise as a hierarchical level to develop norms in the Internet use of their children. These results warranted the design of the established program.
\end{abstract}

Keywords: instructional programmes; father; internet; children. 


\section{Introducción}

En el presente artículo, se refiere al uso del Internet desde una óptica de transmisor de información y conocimientos para convertirse en el orientador de los mecanismos que ha de adoptar los padres y representantes en las diversas situaciones de aprendizaje de sus hijos. Finalmente, se espera que sea del máximo provecho del lector toda esta información, a los fines de conocer y entender profundamente la tecnología de la información (el internet), como el arte y la ciencia de ayudar a los padres y representantes a aprender, orientar y guiar en el uso adecuado de esta herramienta tecnológica para sus hijos, tomando en consideración que todo ser humano desde su nacimiento hasta el final de sus días, tiene el derecho a su crecimiento y satisfacción personal generado por el aprendizaje tecnológico.

De allí que, este aprendizaje en la era de la tecnología exige cambios en el sistema educativo venezolano, encargado de dirigir sus políticas educativas para lograr la formación que se quiere en el desarrollo del ser, conocer, hacer y convivir. En este sentido, el objetivo de la educación debe ser encauzado a los diferentes niveles y modalidades, para así lograr la transformación de un ciudadano que necesita y exige nuestra sociedad actual, es decir, que esté en sintonía con los nuevos avances tecnológicos y científicos de nuestra era.

Nuestra sociedad venezolana, ha sufrido cambios en materia de legislación, y es que las leyes que apoyan al niño y al adolescente en lo que respecta a la protección-autoridad por parte del estado y de los padres, señalan que debe existir una permanente vigilancia y control tanto de los padres como de los propietarios de las salas de internet, video juegos y otros equipos multimedia, para evitar la exposición de peligros que atenten contra la integridad de los niños/as y adolescentes.

En ese sentido, las recientes investigaciones sobre el uso adecuado de la tecnología de la información realizadas por expertos en diferentes partes del 
mundo, y del país, para conocer los efectos que ejercen en los niños/as y adolescentes el uso del Internet, arrojan resultados alarmantes, en cuanto a la cantidad de horas que son empleadas, la falta de normas por parte de los padres hacia estos, el bajo índice académico, la falta de sociabilidad, y la agresividad en las diferentes instituciones educativas de educación sobre todo en la Tercera Etapa de Educación Básica.

Por lo tanto, es conveniente acotar que la autoridad de la familia es el escenario de la comunicación. Becerril (2006a), expone: "la autoridad de los padres a cambiado influenciada por el uso de nuevas tecnologías" (pág. 12). Antes era el padre quien enseñaba al hijo ahora encontramos otra situación, por primera vez los hijos se han apoderado de la estructura del poder. De modo tal, que en este ámbito se alteran las relaciones de los padres con los hijos, porque ahora son los hijos quienes toman la autoridad y enseñan a los padres.

De allí que, se debe recordar que la familia, es una institución que cumple una importante función social, en la trasmisión de información, creencias, valores y actitudes los cuales a la vez juegan un papel decisivo en el desarrollo de sus integrantes. Por lo tanto, el papel de este organismo en la sociedad ha exigido que la familia contribuya y facilite todos los materiales necesarios para el desarrollo y el bienestar de sus miembros.

De lo anterior se deduce, que la familia desempeña un rol en la educación formal e informal, es un espacio donde son absorbidos los valores y donde se profundizan los lazos de amistad. Al respecto, (Ferrari y Kalustron, 2002), expresan lo siguiente: "En el interior de la familia se construyen los límites entre las generaciones y son observados los valores culturales" (pág. 10). Es así como la familia viene a ser el escenario donde se lleva a cabo el desarrollo de la identidad y el proceso de socialización del individuo; por lo cual se debe destacar que cuando esta es saludable posibilita a la institución familiar cumplir exitosamente con los objetivos y funciones que históricamente y socialmente se le han asignado. 
Por otro parte, no se pude negar que las familias atraviesan dificultades que deben ser afrontadas bajo el ejercicio del binomio autoridad-afecto. Para Bianco (2000a), refiere lo siguiente: "Este binomio constituye, la base del funcionamiento operativo de la familia" (pág. 32). Por cual se deben unir estos dos elementos autoridad-afecto. Siendo el primer elemento del binomio temas de interés en este estudio, basado en el principio de autoridad referido por Bianco (2000b), como "la base o fundamento sobre la cual se apoya una idea y la autoridad como la persona que se reviste de poder, mando o magistratura este será el crédito o la fe que se le deposite a alguien" (pág. 12).

Además, el mismo autor puntualiza que los hijos en ningún momento ejercen la autoridad, dicha facultad está concebida a los padres por derecho propio, existiendo una base o fundamento para ello, es un derecho que se adquiere al convertirse en padres. Asimismo, Bianco (2000c), expresa lo siguiente: "la autoridad no se negocia" (pág. 32). En atención a lo planteado, es importante que los padres se involucren con sus hijos y ejerzan la autoridad desde que estos son pequeños, para que la misma se mantenga y cuando ingresen a la educación secundaria que es su momento de adolescencia se mantenga una buena relación con los padres, porque ellos necesitan la misma cantidad de atención y amor que cuando eran pequeños.

Cabe destacar que, si bien es cierto que el conocimiento proporciona poder para el saber, discernir, escoger alternativas razonables, no se debe quebrantar la autoridad de los padres para canalizar y establecer normas en su hogar para el uso de la tecnología, en especial el uso del internet dentro y fuera del hogar, debido a la importancia que tiene la autoridad en el ámbito familiar.

En este sentido, Becerril (2006b), ratifica que se debe tener cuidado con la dependencia que adquieren los hijos en el uso de las nuevas tecnologías advirtiendo los peligros que corren los padres al invertirse la autoridad en el 
ámbito familiar, porque atenta contra la integridad y la funcionabilidad que se le ha asignado a la institución familiar.

La situación antes considerada es una problemática a nivel nacional. De acuerdo con fuentes emanadas de la zona metropolitana de Caracas en un estudio de la agencia de noticias periodismo a favor de la niñez y la adolescencia (PANA) en el año 2006 con el apoyo de las naciones unidas para la infancia (UNICEF), comprobaron que los usuarios más jóvenes adolescentes entre 12 y 17 años y entre 18 y 24 años, representan el $31 \%$ y el $30 \%$ del total de ellos de donde el $37 \%$ cursan secundaria.

Asimismo, según Técnicas Digitales (2006), el acceso general de la población al internet recurriendo a los cybers, $65 \%$, en casa $13 \%$, trabajo $18 \%$, centro de educación, $7 \%$, infocentro $4 \%$. A través de una computadora y de una línea de teléfono los niños, niñas y adolescentes tienen acceso a una cantidad infinita de información y tienen oportunidad para la interacción.

De las evidencias anteriores señalada, se puede decir que existe peligros y riesgos reales para el adolescente que no tiene supervisión, de hecho, la capacidad que tienen los jóvenes de ir de un lado a otro con solo presionar un clic de la computadora le genera al adolescente impulsividad, adicción y la pronta gratificación. En tal sentido, comenta el último autor mencionado, que se puede generar adicción al internet en niños, niñas y adolescentes provocando graves trastornos psicopatológicos como depresión, crisis de ansiedad, conductas antisociales o no operativas y en casos extremos actitudes violentas.

Lo anterior expuesto, el estado Lara, no escapa a esta problemática, de acuerdo con declaraciones de la dirección del Consejo Estadal de Derechos del Niño, Niña y Adolescente (CEDNA, 2007), actualmente llamado Instituto Autónomo Nacional de Derechos de Niños, Niñas y Adolescentes (IDENA), reportaron adicción de los jóvenes a los cybers. Por el peligro que reviste la situación planteada refiere Alfonzo (2007), los padres deben impedir que sus 
hijos se mantengan mucho tiempo solo frente a las pantallas de Internet, también es recomendable prohibirles a los adolescentes el uso de videojuegos de acción porque contienen demasiada violencia y esto genera insensibilidad, por lo que se recomienda una comunicación constante con los mismos, brindándoles afecto, amor comprensión y respeto para alejarlos de los complejos y agresiones.

Por consiguiente, la mejor forma de actuar es tomar en cuenta las consideraciones planteadas con respecto al uso del Internet mediante el establecimiento de normas entendiéndose este término, según (Bianco, 1998a), "norma significa simplemente regla (pág. 23), para el Diccionario de la Enciclopedia de la Psicología (2000a), define la norma como regla que estructuran las relaciones interpersonales". Por eso es importante destacar que los padres deben negociar las normas con sus hijos para el uso operativo del internet con el objetivo de mejorar las relaciones interpersonales y el comportamiento social de los adolescentes.

Ante la situación descrita anteriormente, los padres de los adolescentes de la Unidad Educativa "Departamento Libertador" ubicada en el oeste de la ciudad, Parroquia Unión, Municipio Iribarren. Quienes provienen de diferentes sectores detectándose un mínimo 6 cybers adyacentes a estos, actúan como una situación estimulo en los adolescentes. De acuerdo a las opiniones de los padres la problemática se presenta por el cambio de conducta observado en sus hijos, recibiendo quejas de los profesores por escaparse de clases, manifiesta también que se ausentan del hogar durante el día y la noche sin pedir permiso o sin notificar, lo cual reconocen que se ha escapado el control de su autoridad con respecto a la aplicación de las normas en el uso del Internet en los cybers, manifestando que no poseen herramientas de control ni una orientación adecuada para rescatar su autoridad y las normas en el hogar. 
Las evidencias anteriores han traído como consecuencia conflictos en el hogar alterando la paz familiar, si como también bajo rendimiento académico en los hijos, perdida de dinero, cambio de hábitos alimenticios, ausencia de prácticas deportivas, visto de esta forma y de continuar esta problemática se puede derivar en estos estudiantes conductas de dependencia al uso del Internet, marcada vinculación con los compañeros adictos al mismo, deserción escolar, delincuencia, entre otros.

\section{Teoría y Conceptos}

\subsection{Conductas Operativas de los Padres hacia sus Hijos (as).}

Durante muchos años, la conducta humana ha sido objeto de estudio, por lo que viene a ser el tópico que más inquieta a los especialistas en materia conductual, tomando como punto de partida al hombre como regente del planeta tierra, las reacciones del ser humano puede ser innata o producto del aprendizaje, el cual está determinada por los principios experimentales, que ya han sido objeto de estudio previos, que han permitido establecer las herramientas para tratar las diversas dificultades en materia de conducta aprendida. De allí que, las conductas denominadas innatas o incondicionadas, tienen su origen en la acción determinante de los centros y vías que operan en el sistema nervioso, el sistema nervioso es el operador principal de las acciones sensoriales y motoras que observamos durante los primeros meses de vida de un individuo, Moles (2000a).

En efecto, las conductas que manifiesta un individuo en su cotidianidad pueden ser objeto de un condicionamiento operativo, o ser parte de un modelamiento por parte de su entorno, lo que llamarían los expertos conductas aprendidas, en este contexto juega un papel importante la voluntad del sujeto, el determina si aprende o no la conducta. En este escenario jugará un papel determinante los factores ambientales que pueden ser el detonante que logre la fijación de una conducta no deseada, conjugando la acción que puede 
ejercer el sistema nervioso central como centro de la voluntad del hombre, todos estos estímulos complicarían más la conducta que está por aprenderse según lo establece, Moles (2000b), para abordar las conductas operativas, que es la motivación inicial de esta investigación, es importante destacar, los comportamientos no operativos de los padres, haciendo referencia aquellas conductas donde ellos manifiestan en sus hijos y que producen un efecto negativo en el desarrollo socio afectivo de la generación de relevo. Entre los más comunes destacan:

Irrespetar los sentimientos del niño (por ejemplo, prohibiéndole al niño que manifiesta su desenfado con una situación que lo molesta, castigarlos si se irritan); Ignorar las emociones y sentimientos del niño, los padres en su ignorancia piensan que sus hijos son sistemas cerrados, es decir que no se alimentan de ese entorno afectivo, afectándolo en un gran porcentaje a los padres que no entienden que sus hijos son diversas individualidades.

Ahora bien, un padre responsable valora los sentimientos de sus hijos, busca las herramientas idóneas, para resolver el mundo afectivo que se encuentran, sin embargo a veces comente errores en plantear soluciones emocionales alternativas, y piensan que puede controlar y manejar sus emociones inadecuadas, (por ejemplo, pegándoles) que viene hacer la otra cara de la moneda, de igual forma la existencia de tolerancia exagerada, los padres que por principios le permiten todo a sus hijos, pierden la ocasión de enseñarle estrategias conductuales para manejar asertivamente sus emociones.

Por consiguiente, los padres deben aprender a valorar las emociones de sus hijos. Partiendo de sus individualidades como un mundo único que puede ser enriquecido con un verdadero amor y respecto, direccionando una existencia sana y feliz. Es relevante destacar que los padres con principios y prejuicios muy rígidos ante la vida no toleran que sus hijos manifiesten ninguna 
actitud emocional que vaya en contra del paradigma que ellos practican, convirtiendo la existencia de la Sobreprotección.

Sobre la base de las ideas expuestas es importante tomar en cuenta ciertos elementos como lo son: Los Miedos, que son considerados una manera de conducta en el hombre, que pueden ser transmitidos de los padres a los hijos, aunque los padres crean que no lo han manifestado abiertamente ante ellos, cundo se habla de miedos se está abordando las emociones más fuertes.

Asimismo, la Agresividad, corresponde a la violencia física o verbal considerada una conducta negativa que puede marcar profundamente la vida psíquica del menor a tal punto que tendremos a futuro un adulto violento y desadaptado que no podrá vivir en pareja por la manera como fue marcado por sus padres. Con respecto al Pesimismo, los padres pesimistas programan a sus hijos para resignarse frente a los fracasos y dificultades. Aquí el mensaje es hagan lo que hagan, no tienen capacidades para influir en los acontecimientos de su vida, los éxitos son casuales y los fracasos incontrolables

En este mismo contexto, según Ryan (2000), expresa que: este fenómeno conductual opera cuando hay relaciones de dependencia entre las personas, padres e hijos, profesores y alumnos. De allí que, las expectativas se pueden considerar como una energía que se transmite por creencia en una autoridad dándole todo el crédito con un alto porcentaje de que suceda un hecho o acontecimiento.

Con respecto a los padres, lo operativo es tener claro, las expectativas en lo que respecta a conducta que esperan de sus hijos y unas relaciones familiares asertivas para unas relaciones armoniosas. Abordando las expectativas de los padres a veces su discurso es incongruente con lo que predican: debido a que los padres actúan de forma contradictoria. Por ejemplo, se le dice al hijo que no consuma bebidas alcohólicas porque es mala para la 
salud, pero los padres son consumidores de asiduos de alcohol, el padre que le dice a su hijo que tiene que estudiar para el futuro, pero lo premia aun cuando ha reprobado varios semestres en la universidad.

En cuanto a las relaciones familiares afectivas, como todas las relaciones humanas, las relaciones con la familia son recíprocas y deben alimentarse. Por lo tanto, los padres requieren tener clara la importancia de las mismas, apoyándose para ello en el ejercicio de la autoridad y el afecto.

De igual manera el Respeto: como valor es considerado una cualidad que debe ser innata en el hombre, debe ser la motivación que determina el quehacer cotidiano de toda acción humana. En su estado original el respeto crea una fuerza interior en el hombre para tener la fe necesaria en su propio ser, para elevarse en su paz interior para acometer solo actos nobles en función del entorno familiar

Un hombre que tiene como fundamento el respeto, tiene la capacidad de crea un ambiente de respeto, proyectando a las diferentes instituciones ese valor como fundamento de vida, y se evitan conductas no operativas en el funcionamiento de instituciones del estado, hay que destacar la importancia de agregar al respeto el valor de la humildad como filosofía de vida estaríamos hablando de una sociedad producto de un imaginario que sería el estado ideal de una sociedad perfecta.

Con referencia al Afecto según Ryan (ob.cit), se puede decir, que es un proceso de interacción social que se da entre dos o más personas, es algo que fluye y se traslada de una persona a otra, es diferente de la emoción pues la emoción es una respuesta individual interna y no requiere la interacción con otro. Por lo tanto, brindar afecto requiere de un esfuerzo y una intencionalidad, pues las personas se esmeran en demostrar ese afecto y fomentan conductas para ello.

Por último, el Binomio Autoridad-afecto: es necesario redefinir quien va a ejercer la autoridad. En este caso será la madre, como cabeza de la familia. 
Esto no quiere decir que el padre no tenga ni voz ni voto, y que carezca de todo derecho a opinar sobre la educación de los hijos; pero, en última instancia, la persona que lleva la responsabilidad de hacer cumplir la autoridad es la madre. A su vez, el ejercicio del afecto le va a permitir establecer y mantener el nivel de notificación esencial para el cumplimiento de sus deberes, el disfrute de sus derechos y alcanzar una adecuada comunicación entre ellos.

Por lo tanto, la comunicación es considerada esencia en el proceso transformador de las conductas; significa compartir, abrirse en uno al otro, expresar sus ideas, pensamientos y deseos, es decir, entenderse con sus semejantes. Para Moles (2000c), es considerada como la "acción de transmitir una impresión, ideas, razonamiento o afecto de un individuo a otro" (pág. 159). $Y$ es que actualmente, los niños y niñas necesitan ser escuchados, ya que escuchar no es igual que oír las palabras, sino que va más allá, pues se trata de atender a la persona que está detrás de la palabra.

\subsection{Padre}

La palabra Padre según el Diccionario $A B C$ es considerada un término por demás de popular en nuestro idioma, ya que lo empleamos extendidamente y en diversos ámbitos y situaciones. Uno de los usos más habituales de la palabra es para referir a aquel ser humano de género masculino que tras haber obtenido descendencia directa, es decir, hijos, se convierte en el progenitor de éstos, antecediéndolos.

Ahora bien, cabe destacarse que también se llama padre a quien cumple el rol de cuidado, protección y educación de sus hijos, aunque entre ambos no exista una conexión biológica. En nuestra cultura el rol del padre resulta ser fundamental para el desarrollo del individuo.

\subsection{Hijo}

Según Saramago (2017), Hijo es un ser que Dios nos prestó para hacer 
un curso intensivo de cómo amar a alguien más que a nosotros mismos, de cómo cambiar nuestros peores defectos para darles los mejores ejemplos y, de nosotros, aprender a tener coraje. Ser madre o padre es el mayor acto de coraje que alguien pueda tener, porque es exponerse a todo tipo de dolor, principalmente de la incertidumbre de estar actuando correctamente y del miedo a perder algo tan amado.

Entonces, el concepto de hijo se encuentra estrechamente vinculado al de padres, es decir, sin padres no habría hijos y viceversa. Mientras tanto a aquella persona que no tiene padres porque lo abandonaron o porque murieron se lo llama huérfano. Ahora bien, debemos destacar asimismo que no solamente hijo será aquel que fue procreado por sus padres, es decir, que lo concibieron a partir de una relación sexual, sino que también será hijo aquel que adopta una pareja y que es anotado legalmente como hijo. Normalmente se le distingue como hijo adoptivo, aunque es un hijo como cualquier otro.

\subsection{Programa}

El concepto de programa (término derivado del latín programma que, a su vez, tiene su origen en un vocablo griego) posee múltiples acepciones. Puede ser entendido como el anticipo de lo que se planea realizar en algún ámbito o circunstancia; el temario que se ofrece para un discurso; la presentación y organización de las materias de un cierto curso o asignatura; y la descripción de las características o etapas en que se organizan determinados actos o espectáculos artísticos.

\subsection{Establecimiento de Normas}

Unos de los aspectos más relevantes en el seno familiar es el establecimiento de normas para la actuación de sus miembros, en tal sentido, amerita ser conceptualizado, bajo la óptica de este estudio. Para Bianco (1998b), Normas significa simplemente "regla" (pág. 23), para el Diccionario 
de la Enciclopedia de la Psicología (2000b), la define como "Reglas que estructuran las relaciones interpersonales y los comportamientos sociales de las personas" (pág. 143). Por otra parte, el Centro de investigación Psiquiátrica, psicológica y sexológicas de Venezuela (1994), en su reglamento lo define como "Un patrón de comparación con el cual se juzga el comportamiento, por tanto, poseen el mandato de otorgar aprobación o repulsa" (pág. 24).

Además, presenta una serie de características reseñados por el primer autor: a) se caracterizan por ser regional, es decir que tiene vigencia en localidades específicas, en este caso en los hogares de los padres de adolescentes en estudio, pudiendo o no generalizarse a otros grupos 0 regiones. b) la segunda caracterización es que no está abierto a la investigación, lo que significa que es derivado de decisiones individuales 0 grupales (padres, hijos, adherentes) los cuales se aceptan como una realidad debiendo de ser respetados y por último son de carácter subjetivo, las normas pueden variar de una región a otra, pero su carácter de verdad vinculante se aplicara sin necesidad de aplicar alguna técnica de carácter científico.

Es evidente que las normas o reglas deben existir, al respecto refiere Moles (2000d), "La regla predispone a un hábito y la repetición del mismo forma una creencia. De tal modo que el mantenimiento de la creencia mantiene una regla social y a su vez esta condiciona al habito retroalimentación circular" (pág. 212). Por tanto, en concordancia con el autor ante mencionado el manejo adecuado de las normas permite alcanzar la autonomía, debido que es considerado un largo proceso, la cual se debe dar en etapas, considerado que existen límites.

Por tanto, es importante destacar los requisitos para elaborarlas y sean eficaces las normas o reglas en el ambiente familiar, es necesario que ellas deban ser pocas y precisas, además de fáciles de entender, asimismo deben ser justas, que tengan consecuencias proporcionadas, donde exista un punto 
de bondad. Por otra parte, tienen que ser coherentes, si hay varios hermanos, las reglas deben funcionar para todos, también deben ser consistentes, si se ha llegado a un acuerdo, el adolescente debe cumplir; que no sea cuando a él le apetezca.

\subsection{Reglamento de los padres en el uso de Internet}

En el ejercicio de su autoridad, los padres pueden aplicar una serie de premios y castigos para el uso de Internet, ante el cumplimiento o incumplimiento de las normas de los adolescentes hacia el uso del mismo. Las conductas según Bianco (1998c), se modifican de acuerdo con las consecuencias, si estas son agradables, aumentan la posibilidad y emisión de aquellas. Si por el contrario son aversivas o desagradables disminuyen la probabilidad y emisión de las mismas.

En este sentido, existen premios y castigos para modificar o instaurar o conductas en los adolescentes en el uso del internet. Según Bianco (1998d), existen diferentes reforzadores para la adquisición de nuevas conductas, premios, reforzamientos positivos, reforzamientos negativos, moldeamientos por aproximaciones sucesivas e imitación. Los premios son reforzadores positivos que pueden lograr cambios significativos para lograr una conducta deseada, por eso es importante cultivar la alabanza, atención y el cariño como herramientas para un desarrollo personal integral.

En relación con el reforzamiento negativo, el mismo autor señala que consiste en el aumento en la probabilidad de que una respuesta se presente como consecuencia de la omisión de un estímulo u objeto. Por ejemplo: el niño que obtiene altas calificaciones en el colegio porque de no hacerlo le prohibirán el uso del Internet por una semana.

En cuanto al modelamiento por aproximaciones sucesivas, el mismo autor plantea que los niños y adolescentes aprenden por medio de la observación e imitación de las conductas de otros modelos y de las 
consecuencias de éstas. Por ejemplo, el padre que desea que sus hijos cumplan con los acuerdos establecidos y respeten el horario del Internet, no chatear con desconocidos, no abrir páginas pornográficas, entre otras, al mismo tiempo se debe considerar el castigo como mecanismo que facilite al niño el camino de la honradez, la obediencia y la aplicación, para hacer de él un hombre con principios, éticos y moral. El castigo más que para enmendar la culpa cometida debe servir para la corrección para esto es necesario que el niño reconozca la falta, y lo justo del castigo.

En cuanto al tiempo fuera, el autor señala que este procedimiento se aplica cuando el reforzador que mantiene la conducta no se puede suspender. Consiste en retirar al sujeto de la situación en la cual emite la conducta que se desea suprimir. Por ejemplo, el adolescente que es retirado del lugar donde se encuentra el computador por un periodo de tiempo porque es sorprendido chateando con desconocidos.

En relación con el reforzamiento de conductas incompatibles, es un procedimiento para disminuir la ocurrencia de conductas no operativas sin necesidad de utilizar estimulación aversiva. Por ejemplo, el adolescente que excede las horas establecidas en el uso del Internet, la madre le ofrece dinero para salir a pasear por cada media hora que disminuya el uso del Internet.

\section{Metodología}

La presente investigación fue de tipo proyectiva, apoyado en una investigación de campo de naturaleza descriptiva. La Población y muestra fue constituido por setenta (70) individuos (padres), considerando que en esta oportunidad los elementos del conjunto son los padres que conforman la tercera etapa 3er y 4to año de la Unidad Educativa Departamento Libertador Parroquia Unión Barquisimeto Estado Lara. Se diseñó un cuestionario, conformado por veintinueve (29) ítems de alternativas de respuestas si-o-no, midiendo tres (3) dimensiones, Autoridad-Afecto-Uso del Internet en donde los 
encuestados marcaron con una $(\mathrm{x})$ la alternativa que le correspondió a cada caso. Asimismo, se cumplió con el desarrollo de las fases que comprende a la investigación proyectiva descriptiva, que son:

Fase I. En esta fase se realizó el diagnóstico dirigido a la recolección de información, para tener unos panoramas completos de la situación estudiados. Para tal fin se aplicó un cuestionario a los padres sujetos de estudio. Luego se procedió dar cumplimiento a la Fase II. Con respecto al Diseño del Programa. Una vez recopilado y analizado los datos correspondientes al diagnóstico se procedió a diseñar una propuesta.

Finalmente se ejecutó la Fase III, que consistió en la Validación del Programa con el propósito de valorar el grado de representatividad del material, claridad y congruencia de la redacción, en el programa y que debe cumplir con los objetivos propuestos en dicho programa.

Con respecto a las Técnicas de Análisis de Datos: La información recabada por medio del instrumento fue tabulada en base a la frecuencia y porcentajes de las respuestas de los padres a través del programa informativo de Excel, presentando los resultados obtenidos en cuadros estadísticos en base a cada dimensión de las variables en estudio. Luego se calcularon los promedios de cada nivel de la escala utilizada en los mismos. Es importante destacar que los cuadros estadísticos permitieron la codificación, organización y tabulación de las respuestas a través de un análisis sencillo y confiable de los datos aportados por los sujetos de estudio. Es relevante acotar que el programa aplicado reúne las condiciones y la fundamentación del modelo Gagné. A continuación, se presenta cuadro y grafico obtenido de la aplicación del instrumento. 
Cuadro 1. Valores en frecuencia y porcentajes de la respuesta de los sujetos encuestados según el indicador: Ser autoridad y la Dimensión Autoridad.

\begin{tabular}{ccccc}
\hline \multirow{2}{*}{ Ítem } & \multicolumn{2}{c}{$\mathbf{S i}$} & \multicolumn{2}{c}{ No } \\
\cline { 2 - 5 } & $\mathrm{F}$ & $\%$ & $\mathrm{~F}$ & $\%$ \\
\hline 1 & 49 & 81,6 & 11 & 18,3 \\
\hline 2 & 50 & 83,33 & 10 & 16,66 \\
\hline 3 & 47 & 78,33 & 13 & 21,66 \\
\hline Total & $\mathbf{4 8}$ & $\mathbf{8 1 , 0 8}$ & $\mathbf{1 1 , 3 3}$ & $\mathbf{1 8 , 8 7}$ \\
\hline
\end{tabular}

Fuente: La Autora (2009).

Gráfico 1. Valores en porcentajes de la respuesta de los sujetos encuestados según el indicador: Ser autoridad y la Dimensión Autoridad.

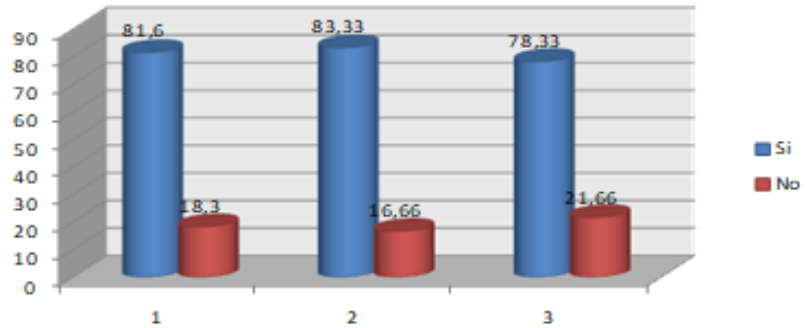

Fuente: La Autora (2009).

\section{Resultados}

Los resultados obtenidos demuestran que la propuesta de un programa dirigido a los padres para el uso del internet de sus hijos, es una necesidad detectada en los padres o madres que son representantes de los estudiantes de la Unidad Educativa Departamento Libertador, ubicada en la Parroquia Unión, Barquisimeto Estado Lara, considerando que es una alternativa operativa para la solución de los problemas de estos padres que necesitan ejercer su autoridad operativamente e implementar normas a sus hijos en el uso del Internet. 


\section{Conclusión}

Por el diagnóstico obtenido en la aplicación del instrumento a los padres encuestados hacia sus hijos para el establecimiento de normas en el uso del Internet, se pudo evidenciar que no establecen normas de forma sistemática a sus hijos, pues estas se establecen por parte de los padres, pero no se canalizan y no se discuten y planifican en reunión familiar, es decir no existe el binomio Autoridad-Afecto, sino más bien autoritarismo.

Por otra parte, no se acostumbra a dar expresiones de amor entre los padres e hijos, son pocos los padres que acostumbran a dar un beso y un abrazo a sus hijos y viceversa. Sin embargo, la aplicación del castigo no es operativa esto ratifica que no se realizan las reuniones familiares para discutir las normas y reglas a cumplir en el uso del Internet.

Por lo tanto, existe la necesidad de diseñar de un programa dirigido a los padres en el uso del Internet para sus hijos, porque representa una alternativa de solución para los padres ya que permiten que puedan ejercer el binomio autoridad-afecto, de forma operativa y se puedan establecer normas sin ningún tipo de conflicto familiar.

\section{Recomendaciones}

- Solicitar a la zona educativa un trabajo coordinado con el Instituto Autónomo Consejo Nacional de Derechos de Niños, Niñas y Adolescentes (IDENA), para realizar un seguimiento a los diferentes cybers de la Parroquia Unión.

- Realizar un trabajo conjunto con la Alcaldía y la Gobernación, para solicitar el cumplimiento de las normas que rigen el uso del Internet.

- Profundización sobre el tema investigado, para que sirva como base teórica y metodológica sobre otros trabajos de investigación sobre el uso del Internet. 


\section{Referencias}

Alfonzo, M. (2007). Niños y Adolescentes deben utilizar Internet bajo debida vigilancia de Adultos. [Artículo publicado en El Impulso, noviembre 2007].

Becerril (2006a,b). La tecnología y la autoridad del padre. México: McGrawHill.

Bianco, F. (1998a,b,c,d). Formación y Actitudes del Orientador. (FAO). I. Programa de Postgrado. Centro de Investigaciones Psiquiátricas Psicológicas y Sexológicas de Venezuela.

Bianco, F. (2000a,b,c). Educación para padres. Editorial Centro de Investigaciones Psiquiátricas, Psicológicas y Sexológicas. Caracas, Venezuela.

CEDNA (2007). Consejo Estadal de Derechos del Niño, Niña y Adolescente. Caracas, Venezuela.

Diccionario de la enciclopedia de la psicología (2000a,b). Norma. Barcelona: Océano.

Ferrari y Kalustron, (2002). Asertividad familiar para negociar. Edición, México Editorial: McGraw-Hill.

Moles, J. (2000a,b,c,d). Asesoramiento Clínico. Caracas: Editorial Greco, S.A.

Reglamento del Centro de Investigación Psiquiátrica, Psicológica y Sexológicas de Venezuela (1994). Reglamento. Caracas: Autor.

Ryan R. (2000). Teoría de la autodeterminación y la facilitación de la motivación intrínseca, el desarrollo social y el bienestar. Psicólogo Americano. Nueva York: Plenum.

Saramago J. (2017). Definición de hijo. [ Documento en línea]. Recuperado de: http://www.traslamascara.com/definicion-de-hijo-segun-saramago/

Técnicas Digitales (2006). Peligros y Riesgos en el uso de los cybers. Caracas, Venezuela. 
Maria Viscencia Gil

e-mail: mariagil t@hotmail.com

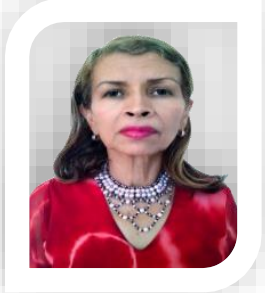

Nacida en Venezuela. Egreso como docente de aula en el año 1979, de la escuela de maestras Miguel José Sanz ejerció en el subsistema de Básica por un lapso de 27 años. Al mismo tiempo curso estudios a distancias en el Mejoramiento Profesional del Magisterio egresando como docente integral mención Ciencias Naturales. Realiza estudios de capacitación en el pedagógico extensión Barquisimeto de 5to nivel. Continua estudios de posgrado en orientación de la conducta en el instituto de psicológica, sexológica de Venezuela, sede Barquisimeto egresando como magister en Orientación de la conducta. Inicia actividades docentes en el instituto tecnológico Antonio José de Sucre Barquisimeto. Recibe capacitación en Investigación Educativa Seguridad industrial en la modalidad de diplomado y en plataforma SAIA. Actualmente cursa estudios de doctorado en ciencia de la educación, culminando la etapa académica y en proceso de tesis. Combina su trabajo del tecnológico con un proyecto motor del programa INCES en un trabajo de atención con jóvenes especiales. Con respecto a la línea de investigación del trabajo de grado doctoral es liderazgo participativo para la transformación de la sociedad, teniendo como fundamento la educación y su transformación en el ámbito educativo.

El contenido de este manuscrito se difunde bajo una Licencia de Creative Commons ReconocimientoNoComercial-Compartirlgual 4.0 Internacional 